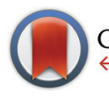

CrossMark

Cite this: Inorg. Chem. Front., 2015, 2, 485

\title{
Correction: 1D chains, 2D networks and 3D interdigitated frameworks of isoorotic acid or 4,4'-bipyridyl and isoorotic acid: syntheses, structures, and sorption properties
}

\author{
Ritesh Haldar, ${ }^{a}$ K. L. Gurunatha, ${ }^{\mathrm{b}}$ Nivedita Sikdar ${ }^{\mathrm{b}}$ and Tapas Kumar Maji*a,b
}

DOI: 10.1039/c5qi90011e

Correction for '1D chains, 2D networks and 3D interdigitated frameworks of isoorotic acid or 4,4'-bipyridyl and isoorotic acid: syntheses, structures, and sorption properties' by Ritesh Haldar et al., Inorg. Chem. Front., 2015, 2, 278-289.

rsc.li/frontiers-inorganic

The authors regret that, in the above paper, the ligand named as 2,4-dihydroxypyrimidine-5-carboxylic acid $\left(\mathrm{H}_{3}\right.$ iso $\left.{ }^{\mathrm{i}}\right)$ (compound 4$)$ should read 2,6-dihydroxypyrimidine-4-carboxylic acid. The structure given in Fig. 6 in the paper is incorrect, and should be modified as given below.

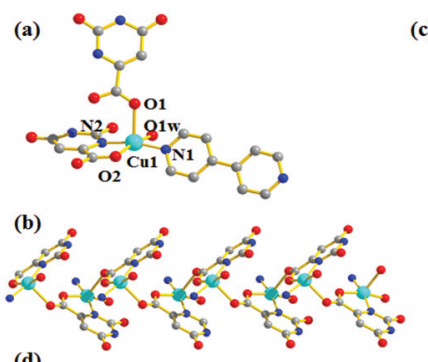

(c)

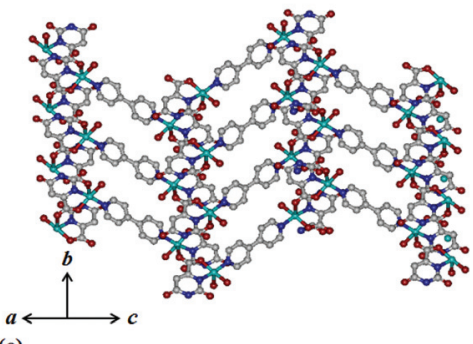

(d)

(e)

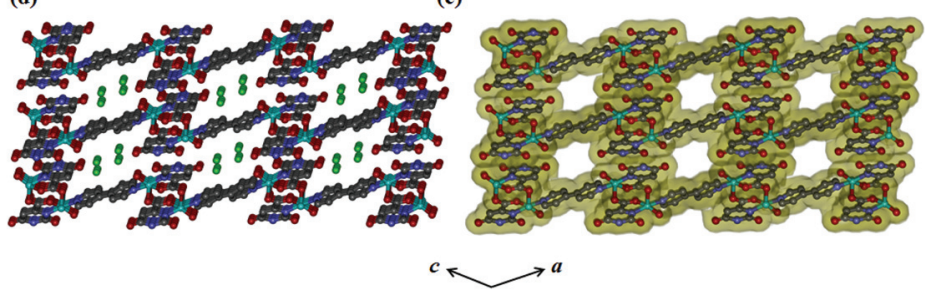

Fig. 6 (a) View of the coordination environment of $\mathrm{Cu}(\mathrm{II})$ in compound $\left\{\left[\mathrm{Cu}\left(\mathrm{Hiso} \mathrm{i}^{\mathrm{i}}\right)\left(4,4^{\prime}-\mathrm{bipy}\right)\left(\mathrm{H}_{2} \mathrm{O}\right)\right]\right\}_{n}$ (4); (b) 1D chain of $[\mathrm{Cu}(\mathrm{Hiso})]_{n}$ formed by a syn-anti carboxylate bridging; (c) view of the 2D sheet; (d) stacking of the 2D sheet along the $b$-axis showing the stairs like shape of a 2D layer; (e) surface added view along the $b$-axis showing 1D supramolecular channels occupied by guest water molecules. $\left(\mathrm{H}_{3}{ }^{\mathrm{is}} \mathrm{i}^{\mathrm{i}}=2,6\right.$-dihydroxypyrimidine-4carboxylic acid; Cyan: $\mathrm{Cu}(\mathrm{II})$, grey: $\mathrm{C}$, red: $\mathrm{O}$, blue: $\mathrm{N}$, and green: guest water molecules).

The Royal Society of Chemistry apologises for these errors and any consequent inconvenience to authors and readers.

${ }^{a}$ Molecular Materials Laboratory, New Chemistry Unit, Jawaharlal Nehru Centre for Advanced Scientific Research, Jakkur, Bangalore, 560064, India ${ }^{b}$ Chemistry and Physics of Materials Unit, Jawaharlal Nehru Centre for Advanced Scientific Research, Jakkur, Bangalore, 560064, India. E-mail: tmaji@jncasr.ac.in; Fax: +91-80 22082766; Tel: +91-80-22082826 